University of Nebraska - Lincoln DigitalCommons@University of Nebraska - Lincoln

CSE Conference and Workshop Papers

Computer Science and Engineering, Department of

$5-2012$

Exploratory Results for a Mission Specialist Interface in Micro Unmanned Aerial Systems

Joshua M. Peschel

Texas A\&M University, peschel@cse.tamu.edu

Brittany A. Duncan

University of Nebraska-Lincoln, bduncan@unl.edu

Robin R. Murphy

Texas Aઐ'M University,murphy@cse.tamu.edu

Follow this and additional works at: http://digitalcommons.unl.edu/cseconfwork

Peschel, Joshua M.; Duncan, Brittany A.; and Murphy, Robin R., "Exploratory Results for a Mission Specialist Interface in Micro Unmanned Aerial Systems" (2012). CSE Conference and Workshop Papers. 245.

http://digitalcommons.unl.edu/cseconfwork/245

This Article is brought to you for free and open access by the Computer Science and Engineering, Department of at DigitalCommons@University of Nebraska - Lincoln. It has been accepted for inclusion in CSE Conference and Workshop Papers by an authorized administrator of

DigitalCommons@University of Nebraska - Lincoln. 


\title{
Exploratory Results for a Mission Specialist Interface in Micro Unmanned Aerial Systems
}

\author{
Joshua M. Peschel, Brittany A. Duncan, and Robin R. Murphy \\ Center for Robot-Assisted Search and Rescue \\ Department of Computer Science and Engineering \\ Texas A\&M University \\ College Station, TX 77843-3112 USA \\ Email: \{peschel, bduncan, murphy\}@ cse.tamu.edu
}

\begin{abstract}
This paper presents a human-robot interaction (HRI) exploratory study of a dedicated Mission Specialist interface for micro unmanned aerial systems (UAS). Current HRI findings from the micro UAS literature suggest that a Mission Specialist role requires a small, mobile, and visual interface that is dedicated and software-based. A literature survey of humanrobot team modeling, human-machine interaction technologies, and interaction principles applicable to micro UAS, resulted in an identified HRI investigation framework, five synthesized design guidelines, and a system architecture for a dedicated Mission Specialist interface. The interface was implemented and evaluated through an exploratory field study involving 16 specialized emergency responders. Observations from the study suggested that with refinements, a dedicated Mission Specialist interface could be a useful tool for future HRI studies to explore role performance in micro UAS.
\end{abstract}

Keywords-User Interface Designs and Usability Evaluations; Human Factors and Evaluation Methodologies; HRI Applications (Search and Rescue).

\section{INTRODUCTION}

This paper presents the human-robot interaction (HRI)informed design, implementation, and exploratory study of a dedicated Mission Specialist interface for micro unmanned aerial systems (UAS) in the CBRNE (Chemical, Biological, Radiological, Nuclear, Explosives) domain. The micro category of UAS is the most accessible technology for civiliansupported CBRNE operations such as fire and rescue [1], law enforcement [2], and civil engineering [3]. Micro UAS are also widely used in military-supported CBRNE operations [4]-[6]. Current legislative trends for UAS integration into the national airspace suggest that micro UAS are in high demand for these domains and will become widely available much faster than any other category of UAS [7].

The Mission Specialist role in micro UAS is uniquely responsible for operating the micro unmanned aerial vehicle (UAV) sensor payload yet current human-robot interface technology only permits duplicate or shared visualization with, and passive interaction through, the Pilot role [8] (Figure 1). Murphy et al. [9] showed three separate human roles (Flight Director, Pilot, and Mission Specialist) are necessary for micro UAS operation in the urban search and rescue (US\&R)

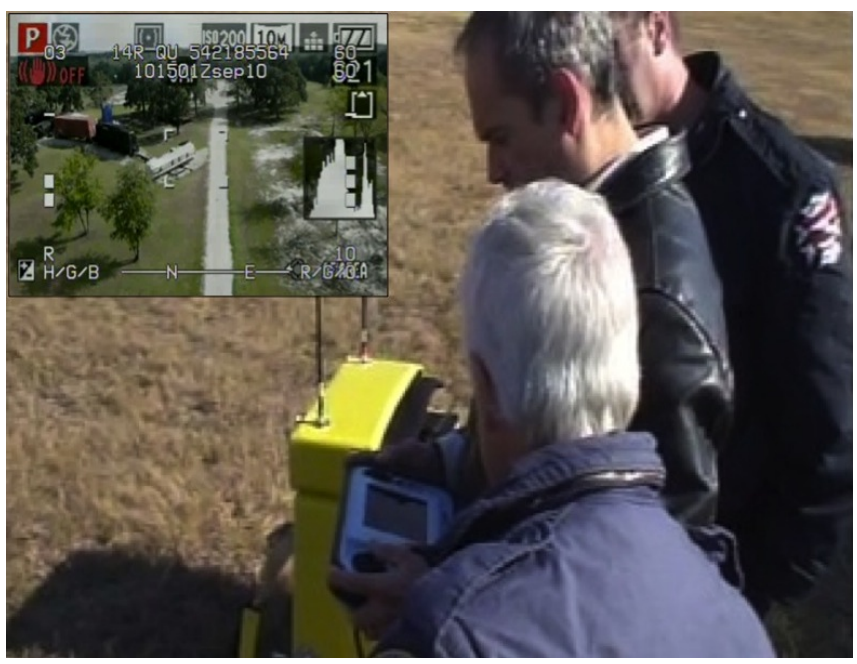

Figure 1. A Micro UAS Mission Specialist (far right) Passively Shares

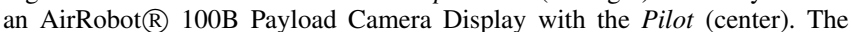
Display (upper left) Contains Numerous Visual Indicators such as Battery Voltage, Flight Time, Distance from Home, etc. that are Important to the Pilot but not the Mission Specialist.

domain and that combining human roles is not preferable. This suggests the current state of practice by the Mission Specialist role is likely in violation of sound human-computer interaction (HCI) principles (i.e., one user to one interface) and that the individual role and overall team performance may be suboptimal for micro UAS [8].

Understanding how a Mission Specialist role should interface with a micro UAV is critical for investigating general HRI in micro UAS, reducing the human-robot crewing ratio, and improving individual human role and team performance. In the context of small unmanned ground vehicles, research has shown high workloads on the decision maker (operator) due to mediation. Because the Mission Specialist is mediated through the Pilot for control of the UAV payload camera, it is anticipated that similar workloads will be experienced in micro UAS. This is important as observations of CBRNE field exercises show that decision makers are not likely to be professional pilots for the near term. For the long term, 
analysis of CBRNE response patterns indicate that even if the Pilot is trained as a specialized emergency responder, there are still remote decision makers (though it is yet unknown if mediation-induced workloads generalize to remote decision makers).

This work provides an HRI investigation framework, five HRI-informed design guidelines, and an implementation for a dedicated Mission Specialist interface for micro UAS based on literature findings. An exploratory study involving 16 specialized emergency responders in the CBRNE domain compares the dedicated interface approach against the Mission Specialist viewing a shared display and directing the Pilot to control the UAV payload camera.

The remainder of the article is organized as follows. Section II serves as a review of research literature for factors associated with Mission Specialist HRI in micro UAS. In Section III, the theoretical approach for this work is given where a Shared Roles Model adapted for a micro UAS is outlined that includes three human team roles (Flight Operations Director, Pilot and Navigator, and Mission Specialist). Section IV describes the implementation of a dedicated Mission Specialist interface for a micro UAS, including the hardware and software specifications. An exploratory field study used to evaluate the dedicated Mission Specialist interface is discussed in Section V. Finally, Section VI presents the conclusions and future directions for this work. The work is expected to i) provide a new tool for HRI researchers to investigate human-robot team performance in micro UAS, ii) serve as a reference document for unmanned system designers and developers and iii) contribute to a better understanding of vulnerabilities in HRI with micro UAS.

\section{RELATED WORK}

This section presents a literature review of related factors associated with understanding the HRI of a Mission Specialist role in a micro UAS. Human-robot team modeling is discussed, with a specific review of Joint Cognitive Systems and the Shared Roles Model for generic unmanned systems. Next, a review of Mission Specialist human-machine interaction (HMI) findings for micro UAS is given. Finally, eight sets of interaction principles are reviewed from both the humancomputer interaction (HCI) and HRI literature.

\section{A. Human-Robot Team Models for Micro UAS}

There are several frameworks from which to consider collaboration modeling in human-robot teams [14]. For the case of a micro UAS, the Shared Roles Model (developed from Social Role Theory and described within the context of a Joint Cognitive System) provides a suitable framework for human-robot team interaction as it was based on empirical unmanned systems studies [15]. The Shared Roles Model is a compromise between two polar opposite approaches the Taskable Agent Model and the Remote Tool Model emphasizing its ability to capture an appropriate balance of robot semiautonomy and the connectivity needs of the human team [15].

1) Joint Cognitive Systems: The Shared Roles Model relies on the viewpoint of a human-robot team operating as a Joint Cognitive System (JCS). As described by Hollnagel and Woods [16], the focus of the JCS is on the co-agency of the participants rather than on the individual participants as distinct components. The what and why are emphasized in a JCS rather than the how. The JCS approach permits less restriction on formalized definition of the cognitive system itself, including functions and processes. This permits an easier description of robots as agents or as artifacts and, more importantly, leads to the idea of the Shared Roles Model [15].

2) Shared Roles Model: The Shared Roles Model is a compromise between the Taskable Agent Model and the Remote Tool Model for describing human-robot teaming. In the case of the Taskable Agent Model, full autonomy of the robot is the goal of the system, with teleoperation being temporary in nature, if necessary at all. On the opposite end of the human-robot model spectrum is the Remote Tool Model. According to the premise of the Remote Tool Model, the robot is essentially devoid of autonomy and used entirely as a tool by the human team. The Shared Roles Model is a hybrid approach that assumes robot semi-autonomy with improved human connectivity for communication [15].

In Murphy and Burke [15], the Shared Roles Model has six different types of primary agents, four shared roles (PilotPlatform Telefactor, Mission Specialist-Payload Telefactor), and two singletons (Safety Officer and Knowledge Worker) (Figure 2). The Mission Specialist role primarily has an egocentric perspective through the UAV that is shared with the Pilot role. The Pilot role primarily has an exocentric perspective of the UAV that is shared with the Mission Specialist role. The Safety Officer and Knowledge Worker roles do not share either perspective. Information transfer can occur between the Pilot and Mission Specialist roles. Communication of mission directives can occur between the Pilot and Knowledge Worker roles. Similarly, transfer of data can occur between the Mission Specialist and Knowledge Worker roles. An important factor to consider in the Shared Roles Model is the potential latency of information transfer, whether it is data from the Mission Specialist role or communication of directives to and from the Pilot role.

\section{B. Human-Machine Interaction in Micro UAS}

Peschel and Murphy [8] surveyed the human team roles and HMI currently used on 10 micro or small UAS humanrobot teams. Three human team role trends were identified (Flight Operations Director, Pilot and Navigator, and Mission Specialist). Findings from [8] suggest that a Mission Specialist role requires a small, mobile, and visual interface that is dedicated and software-based.

1) Micro UAS Human Team Roles: Human team member roles identified in [8] fall into one of three role categories: 


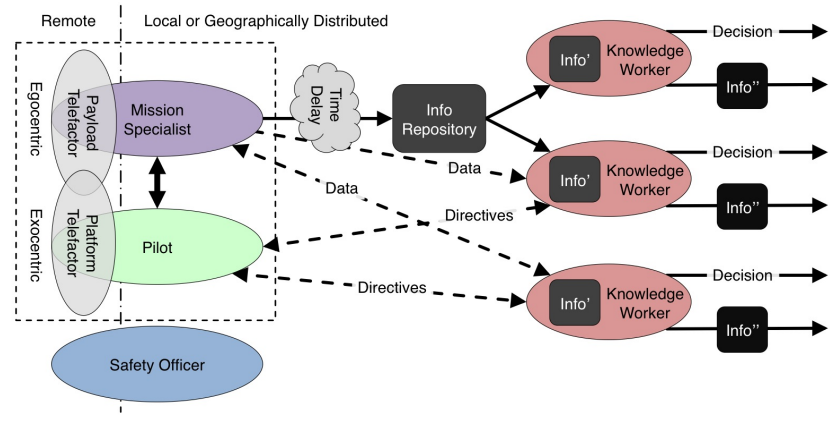

Figure 2. General Illustration of the Shared Roles Model for a Human-Robot Team (Adapted from Murphy and Burke [15]).

Flight Operations Director, Pilot and Navigator, and Mission Specialist. These three role labels represented a synthesis from the literature and were given to describe general role function rather than preferred role identification within any specific UAS.

Flight Operations Director was the role was responsible for proper supervisory planning, coordination, and control of operations that were critical to mission success, especially for a micro UAS human-robot team. Across the micro UAS field studies literature, one or more human team members were found to be responsible for directing the mission.

Pilot and Navigator was the role responsible for piloting the UAV and was common to all micro UAS human-robot teams. However, the degree to which one or more individuals was solely responsible for flight control activity varied; navigation responsibilities were also included as a role responsibility and thus combined with the main function of piloting the UAV.

Mission Specialist was the role responsible for UAV payload sensor control and data acquisition. Micro UAS operational capabilities allow a human-robot team to insert themselves remotely for the main purposes of visual investigation and recording; a role solely responsible for the payload was a commonly identified role.

2) Micro UAS Human-Machine Interaction: Three HMI findings are given in [8] that suggest current Mission Specialist performance in micro UAS may be sub-optimal due to the sharing of a single Pilot and Navigator-oriented interface or a reuse of the Pilot and Navigator interface.

The interaction technology used by the Mission Specialist had the three primary characteristics: mobile, small, and visual. Mobility was observed in all of the interfaces that the Mission Specialist interacted with. Small handheld controllers that could be carried and handled by one individual were the most common form of interaction device. Interactive feedback to the Mission Specialist was visual and took the form of small video displays, graphical menus, and real-time video.

Across the micro UAS literature, the Mission Specialist either shared the same interface with the Pilot and Navigator role, was given a duplicate of the Pilot and Navigator inter- face, or was a passive viewer. No micro UAS in the literature had a dedicated Mission Specialist interface. Given that the Mission Specialist is a unique human team role, a distinct or different modality of HMI technology from that of the Pilot and Navigator would be expected; therefore, existing interfaces, in general, do not support the functionality of the Mission Specialist role.

The responsibility of the Mission Specialist is for data acquisition and, often, interpretation. The possibility for direct manipulation of the imagery for verification, including extracting single static images and a video series for real-time playback while the flight continued to be recorded, appeared present in only one of the ten surveyed micro UAS. These observations in [8] suggest that there is a heavily reliance on hardware-oriented interaction by current Mission Specialists. Current HMI for the Pilot and Navigator-oriented interfaces could be limiting the software capabilities that may improve Mission Specialist performance in micro UAS missions.

\section{Applicable HCI and HRI Principles}

HCI and HRI as design-focused areas in the field of human factors consider issues such as accessibility, awareness, and experience [17]. It is therefore necessary to consider a survey of interaction principles from both HCI and HRI, in order to gain insight and an interaction frame of reference for the investigation of a dedicated Mission Specialist interface.

1) Human-Computer Interaction Principles: At the most fundamental level, HCI is the study of people, computer technology, and the ways in which these two groups influence one another [18]. There have been numerous publications that attempt to present the guidelines that should be used throughout HCI. Not surprisingly, there has not been one universal set of guidelines produced that has been widely adopted. However, from the literature that has been published it is possible to extract salient HCI principles that are applicable to the design of a dedicated Mission Specialist interface. In the following paragraphs, a survey of four fundamentally different approach areas to HCI design principles is presented.

The first set of HCI principles surveyed were from Schneiderman and Plaisant [19] and are based on over thirty years of HCI research, design, and testing across multiple domains. These principles represent a more general, common userapproach to user interface design in HCI. Schniederman and Plaisant refer to their guidelines as the Eight Golden Rules. The next set of HCI principles surveyed were from Sharp et al. [20] and are largely based on the work of Norman [21]. These HCI design principles also represent a general approach to user interface design, but focus specifically on interaction design. Effective visualization of data in a concise format is important for many domain application designs, and especially for the design of a dedicated Mission Specialist interface. Few [22] suggests Thirteen Common Mistakes in Dashboard Design where, by definition, a dashboard is a single-screen display of the most essential information needed to perform a job. 
The final set of HCI principles surveyed were from Endsley et al. [23] and represent an approach to user-centered situation awareness design.

2) Human-Robot Interaction Principles: As with HCI principles, there also does not exist one universally-accepted set of HRI guidelines that have been widely adopted. Consequently, there are no formal HRI guidelines that address the design of a role-based interface for a micro UAS human-robot team. However, a survey of the HRI literature does reveal different investigations for narrow aspects of HRI design such as goaldirected task analysis [24], task structure [25], and metrics of analysis [26]. In the following paragraphs, a survey of four different - but system-relevant - sets of design principles for HRI is presented.

The first set of HRI principles surveyed were from Goodrich and Olsen [27] and are based on previous studies of neglect tolerant autonomy and efficient interfaces. These principles represent a cognitive information processing approach to design in HRI. Goodrich and Olsen refer to their guidelines as the Seven Principles of Efficient Human Robot Interaction. The second set of HRI principles surveyed were from Riley et al. [28]. These HRI principles represent situation awarenessoriented design guidelines for enhancing HRI. The third set of HRI principles surveyed were from Oron-Gilad and Minkov [29]. These principles were developed based on an ethnographic survey of soldiers operating a remotely-operated vehicle, and represent a bottom-up operational perspective. The final set of HRI principles surveyed were from Clarkson and Arkin [13]. The principles were heuristics for the evaluation of HRI systems.

\section{APPROACH}

This section provides the theoretical approach for a dedicated Mission Specialist interface in a micro UAS. A formulation of the Shared Roles Model is presented that includes three formal human team roles (Flight Operations Director, Pilot and Navigator, and Mission Specialist). Five recommended design guidelines are synthesized from the survey of current HCI and HRI principles, which result in a system architecture for a dedicated Mission Specialist interface for a micro UAS.

\section{A. Shared Roles Model for Micro UAS}

To adapt the Shared Roles Model for a micro UAS, the Knowledge Worker role is excluded in order to simplify focus toward the human-robot team. Formal role labels in the model are defined as in Section II-B1: Flight Operations Director, Pilot and Navigator, and Mission Specialist. Payload and platform telefactors, representing egocentric and exocentric perspectives, respectively, remain consistent for this work.

\section{B. Interface Design Guidelines}

Five recommended design guidelines were synthesized based on relevance for a dedicated Mission Specialist inter- face from the survey of HCI and HRI principles. The five recommended design guidelines are as follows.

Guideline 1: Design for Unary Interaction. A unary focus of interaction means that simple, singular aspects of interaction should be considered in all elements of a dedicated Mission Specialist interface design. This may take the form of having the visual display on a single screen, mapping gestures and feedback to control signals in a one-to-one manner, and never being more than one interaction away from any function that the interface is capable of providing.

Guideline 2: Design for Adequate Data Context. Adequate context of any data streams that a dedicated Mission Specialist interface may be capable of handling should be provided. Elements of the interface display and the data contained within them should be clearly identified visually to the user, such as the primary video window, a map, or areas of archived image and video review - there should never exist any data ambiguity during interaction.

Guideline 3: Design for Natural Human Interaction. There are many different modes of interaction for user interfaces graphical, textural, written, haptics, etc. For a dedicated Mission Specialist interface that will be used in field conditions, the impetus of small and mobile form-factoring necessarily dictates users having haptics-based interaction since keyboards and other ancillary devices could be difficult to use.

Guideline 4: Design for Cueing and Communication. In the context of the Shared Roles Model, a human-robot team must be able to appropriately cue and communicate among one another. This should be taken into account for the design of a dedicated Mission Specialist interface and may manifest as subtle alert messages when an image has been captured, or as other visual cues indicating that data is available or there is a message to be communicated within the team.

Guideline 5: Design for Flexibility and Expansion. No good interface design is ever perfect or permanent. Great interface designs allow for flexibility towards the user as new information is discovered about humans and/or domains. Likewise, advances in technology often provide new affordances that can potentially improve existing user interaction. The ability to easily expand functionality will provide longevity to any dedicated Mission Specialist interface design.

\section{System Architecture}

An interface design architecture based on the five design guidelines synthesized for a dedicated Mission Specialist interface is given in Figure 3. The Mission Specialist has two modes of interaction with the dedicated interface: i) viewing of the real-time video data coming from the UAV, and ii) sending control signals to the payload camera. Control signals include camera tilt, zoom, and image capture. Likewise, the UAV transmits real-time video and receives camera control signals. Communication is done wirelessly and through a central network server. Data is stored on the local interface device 


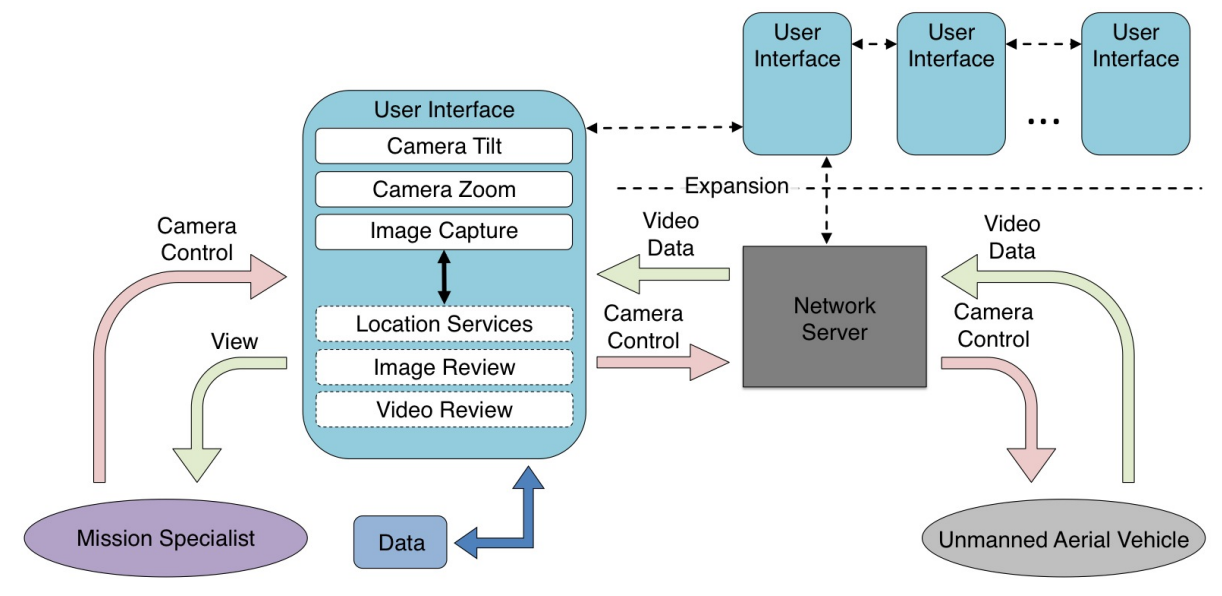

Figure 3. System Architecture for a Dedicated Mission Specialist Interface. The Mission Specialist Has Two Modes of Interaction with the Dedicated Interface: i) Viewing of the Real-Time Video Data Coming from the UAV, and ii) Sending Control Signals to the Payload Camera. Control Signals Include Camera Tilt, Zoom, and Image Capture. (Courtesy of Center for Robot-Assisted Search and Rescue)

(though on many UAV platforms, data can also be stored on the robot). Additional features of location-based services, static image review, and video review could be integrated but will not be evaluated in this study. Additional expansion of the interface technology could include multiple co- or remotelylocated Mission Specialist interfaces networked together to form a common space of interaction, and could possibly include other roles such as the Pilot and Navigator.

\section{IMPLEMENTATION}

The dedicated Mission Specialist interface with system architecture as described in Section III-C was implemented on

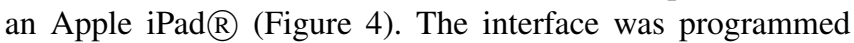
using the Objective-C and standard $\mathrm{C}$ languages. Open-source static $\mathrm{C}$ libraries that provide networking and real-time video processing capabilities were also used.

\section{A. Hardware Platform Description}

The Apple iPad $\AA$ platform was selected for this study due to its mobility, unique multi-touch interface, and ability to provide a usable display in bright outdoor conditions. Apple iPad $囚$ hardware affords three important touch-based gestures that can be customized for the design of an interface: swipes, pinches, and taps. Interaction with the payload camera through Mission Specialist interface consists of swipe up and down gestures to proportionally control the up and down tilt of the camera, in and out pinch gestures to proportionally control the zoom in and zoom out of the camera, and finger tap gestures to control button functionality (e.g., capture an image). Each of these gestures represents a natural and intuitive manner of interaction with the camera.

\section{B. Software Platform Description}

Objective-C is the native programming language of the Apple $\mathrm{iPad} \circledast$; however, much of the non-interface programming (e.g., network connectivity) was accomplished using standard C with open-source static libraries. The Mission Specialist interface communicates wirelessly through an ad hoc network to a dedicated C-based server socket on a laptop computer running Microsoft Windows XP that is connected to the DraganFlyer ${ }^{\mathrm{TM}} \mathrm{X} 6$ base station. This manner of connectivity is a requirement of the DraganFlyer ${ }^{\mathrm{TM}}$ platform. Real-time imagery from the payload camera is streamed to the dedicated Mission Specialist interface using an iOS version of Willow Garage's OpenCV software [30]. Because the server software is written in standard $\mathrm{C}$ and uses OpenCV, the Mission Specialist interface could easily be adapted to other robot platforms that may support a different operating system (e.g., Linux or OSX).

\section{EXPLORATORY STUDY}

This section describes the design overview, participants, measurements, results, and observations from an exploratory study of the dedicated Mission Specialist interface. The study consisted of 16 specialized emergency responders each participating in two different UAS mission trials to visually evaluate and capture images of a simulated train derailment involving hazardous materials. Four types of measurements (performance, biophysical, survey, and audio/video) were recorded for each participant. Observations from the study suggest that a dedicated Mission Specialist interface could be a useful tool for future studies to explore the HRI of individual Mission Specialist role and overall team performance and process for a micro UAS human team.

\section{A. Study Design Overview}

The purpose of the exploratory study was to i) field test the dedicated Mission Specialist interface within the context of actual micro UAS missions and ii) explore the types of measurable interactions exhibited by the Mission Specialist. 
All of the exploratory study mission trials took place at the

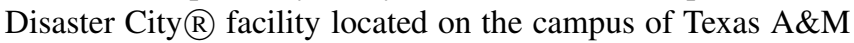
University. The context of the mission was a simulated train derailment involving hazardous materials.

A 16 participant mixed-model study design was developed from the HRI study guidelines in [31]. The number of participants was chosen based on a power analysis to attain power equal to 0.80 in a single group measures design at an alpha equal to 0.05 , given an estimate of mean correlation for repeated measures of 0.80 . Each participant received interface instructions and made two different UAV flights - one per mission trial - with each flight having three pre-defined stationary waypoints and corresponding sets of questions regarding the identification of certain objects (e.g., identify any punctures or ruptures in the tanker truck and capture an image of each you may identify) (Figure 5). The maximum duration of the flights were limited to 7-minutes for consistency and participants were instructed that their goal was to capture images for all of the questions at all of the waypoints within this period of time. The order of the interfaces (conditions) was randomized to counterbalance the study.

During condition 1, half of the participants viewed a mirrored display from the Pilot and Navigator on a laptop and instructed the Pilot and Navigator to control the payload camera and capture images. The laptop was placed on the DraganFlyer $^{\mathrm{TM}}$ X6 base station and adjusted for optimal viewing by each participant. Participants were given a verbal protocol from which they could issue the following camera control instructions to the Pilot and Navigator: tilt camera up, tilt camera down, zoom camera in, zoom camera out, and take photo. The second half of the participants used the dedicated Mission Specialist interface to control the camera and capture images. The Apple iPad $\AA$ was placed on a camera stand that was adjusted for optimal viewing and interaction by each participant. Each participant in condition 2 used the interface they did not have in condition 1. For both flights, participants were given a verbal protocol from which they could issue the following UAV control instructions to the Pilot and Navigator: turn left _-degrees and turn right _-degrees. The verbal protocol for UAV control was made available to participants since the DraganFlyer ${ }^{\mathrm{TM}}$ X6 platform camera mount cannot turn left or right.

The DraganFlyer ${ }^{\mathrm{TM}}$ X6 platform was used during the exploratory study. A professional pilot with extensive experience operating the vehicle served in the Pilot and Navigator role for all of the mission trials. The Pilot and Navigator was instructed to only follow the verbal commands issued by participants regarding camera and UAV control and to not anticipate the intentions of the participants.

\section{B. Participants}

Participants were selected primarily for their experience as specialized emergency responders in coordination with the Texas Engineering Extension Service (TEEX). Detailed

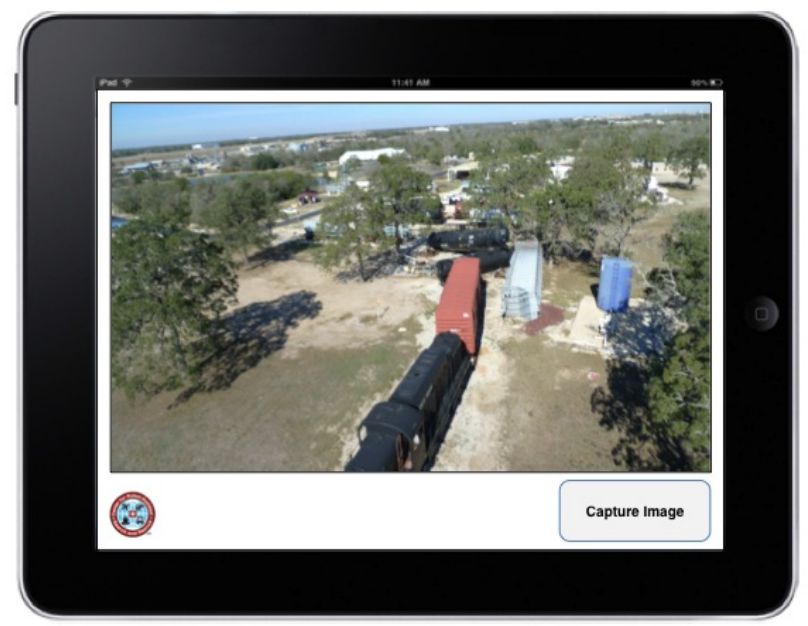

Figure 4. Implementation of the Dedicated Mission Specialist Interface on an Apple iPad $R$. A Captured Image of the Simulated Train Derailment is Shown. The Mission Specialist Swipes (Up and Down) and Pinches (In and Out) Directly on the Video Display to Control the Payload Camera for Tilt (Up and Down) and Zoom (Out and In). Images are Captured by Pressing the Capture Image Button. (Courtesy of Center for Robot-Assisted Search and Rescue)

demographic information about each participant was collected through a pre-assessment survey consisting of 18 questions. Of the 16 participants, 14 were men and 2 were women. Age ranges were: 25-years to 34-years (1 participant), 35years to 44-years (2 participants), 45-years to 54-years (10 participants), and 55-years and older (3 participants). Each of the participants had prior experience with a mobile touchbased device (e.g., Apple iPhone $\AA$ or $i P a d ß)$ ), with frequency of use from several times per week to continuously, for a period of at least one year. The types of interactions participants had with their mobile touch-based devices were: use it as a phone (all participants), check email (15 participants), surf the Internet (14 participants), and play games (7 participants). A majority of the participants had previously used a Tablet PC or other pen-based device (e.g., Palm PDA) but indicated only short-term and/or infrequent usage. There were 9 participants who had prior experience controlling a remote camera either on a robot or through the Internet. Each of the participants, except one, had played a major role in actual search and rescue missions. Only two participants had been involved with a robot-assisted search and rescue mission, which was reported as an exercise.

\section{Measurements}

There were four different types of measurements taken during the exploratory study i) the number of completed tasks, ii) written post-assessment surveys, iii) biophysical measurements, and iv) audio/video recordings. Details for each type of measurement are as follows.

1) Task Completion: Task completion was measured by the number of images a participant captured during each 
mission trial. Images captured when using the dedicated Mission Specialist interface were saved on both the Apple $i P a d \AA$ and the payload camera. When participants used the traditional approach of instructing the Pilot and Navigator to capture an image, resulting images were stored only on the payload camera. All images were time-stamped so they could be correctly associated with the corresponding participant and mission trial.

2) Surveys: Post-assessment surveys to evaluate role empowerment as experienced by the participant in the Mission Specialist role were given. The post-assessment survey consisted of two parts of 10 questions each, and was given after each mission trial (i.e., a post-assessment survey after using the traditional approach, and a post-assessment survey after using the dedicated interface - each with identical questions but in the context of the interface used). Responses addressed confidence and comfort in interface actions as measured on a standard 5-point Likert scale.

3) Biophysical: Participant heart rate was recorded during both mission trials. Biopack BioNomadix $\AA$ sensors were used for wireless recording and were placed on the non-dominant hand of each participant. Prior to the set of mission trials, each participant was asked which hand they would swipe a mobile device with and the sensor was placed on the opposite hand indicated. Active recording status was verified before and throughout each mission trial.

4) Audio and Video Recordings: Four high-definition

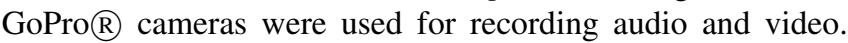
The first camera was mounted on a hard hat that each participant wore to provide a first-person perspective. The second camera was mounted on the stand that held the Apple iPad ( to provide a view of each participant when they interacted with the dedicated Mission Specialist interface. The third camera was mounted to the top of the laptop to provide a view of each participant when they viewed the mirrored display from the Pilot and Navigator. A final camera was placed approximately 20 -feet behind the participants to capture an overall view of the study. Video from the UAV was also recorded. All cameras were calibrated before each mission trial to ensure a correct field of view. Audio and video recorded in this study are currently being coded and analyzed and are not included in the results.

\section{Results}

The dedicated Mission Specialist interface was successfully used in 32 micro UAS mission trials. Measurement data were collected for task completion, levels of stress, and role empowerment. A between $\times$ within analysis of variance (ANOVA) was used to analyze the results from the mixed-factor design study to determine if there was a significant effect between participants and/or within participants, based on the order the participants used the two interface types (traditional approach and dedicated interface) and the interface type used. For each

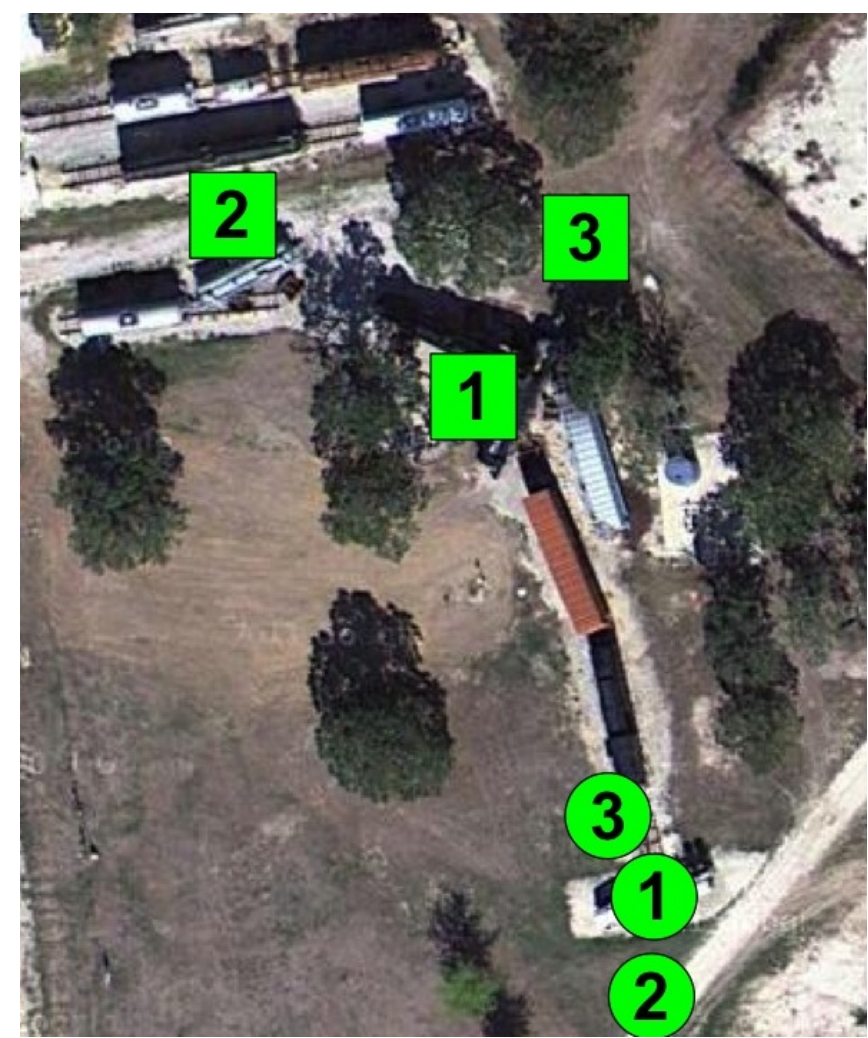

Figure 5. Overhead Map of the Simulated Train Derailment at Disaster

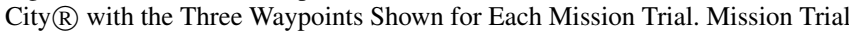
1 Waypoints are Shown as Circles and Mission Trial 2 Waypoints are Shown as Squares. The Numbers Indicate the Three Waypoints in the Ascending Order They Were Visited. (Courtesy of Center for Robot-Assisted Search and Rescue)

of the results, waypoint order was not found to be statistically significant.

1) Number of Completed Tasks: The mixed-model design for number of completed tasks consisted of the between participants factor being the number of captured and the within participants factor being the number of captured images per interface type. These two completed task variables were measured by reviewing the number of captured images on the payload camera for each condition. The interaction of which interface was presented first was also examined for its impact.

The between participants analysis results $(F[1,14]=0.32$, $p=0.58)$ indicated there was not an effect of order in which the interfaces were presented. This result would suggest the participants are likely to perform a similar number of completed tasks regardless of the order in which they used the interfaces. The within participants analysis results $(F[1,14]=12.04$, $p=0.004$ ) suggest there is significant interaction within participants based on the number of images captured on the mission trials and based on the type of interface used.

Image capture statistics were examined to determine the significance of the within participants analysis results. Participants on average captured more images on both mission 
trials when using the traditional approach $(M=6.63, S D=3.01)$ than they did using the dedicated Mission Specialist interface $(M=3.88, S D=2.33)$. On mission trial 1 when participants used the traditional approach, more images were captured $(M=6.75, S D=2.66)$ than when using the dedicated Mission Specialist interface $(M=4.63, S D=2.26)$. On mission trial 2, participants using the traditional approach captured more images $(M=6.50, S D=3.51)$ than when using the dedicated Mission Specialist interface $(M=3.13, S D=2.30)$. These results suggest that mission trial $1(M=4.94, S D=2.56)$ and mission trial $2(M=5.56, S D=1.33)$ had, on average, a difference of less than one captured image. There is also an average of less than a difference of one captured image based on which interface was first used.

2) Level of Stress: The level of stress consisted of the within participant factor being the average heart rate (in beats per minute) per interface type. This biophysical variable was measured by averaging the heart rate data of each participant for each condition. The interaction of which interface was presented first was also examined for its impact. All heart rate results were determined to be non-significant.

3) Role Empowerment: The mixed-model design for role empowerment consisted of the between participants factor being the average 5-point Likert confidence score and the within participants factor being the average 5-point Likert confidence score per interface type. These two survey variables were measured by averaging the post-assessment survey data of each participant for each condition. The interaction of which interface was presented first was also examined for its impact.

The between participants analysis results $(F[1,14]=1.48$, $p=0.24$ ) indicated there was not an effect of order in which the interfaces were presented. This result suggests the participants are likely to experience similar role empowerment regardless of the order in which they used the interfaces. The within participants analysis results $(F[1,14]=14.35, p=0.002)$ suggest there is significant interaction within participants based on reported role empowerment for the mission trials and the order in which the interfaces were presented.

Post-assessment survey statistics were examined to determine the significance of the within participants analysis results. Participants on average reported more role empowerment regardless of mission trial when using the traditional approach $(M=4.53, S D=0.80)$ than they did using the dedicated Mission Specialist interface $(M=3.56, S D=1.07)$. On mission trial 1 when participants used the traditional approach, more role empowerment was reported $(M=4.33, S D=0.96)$ than when using the dedicated Mission Specialist interface $(M=3.84$, $S D=0.97)$. On mission trial 2, participants using the traditional approach reported more role empowerment $(M=4.73$, $S D=0.59$ ) than when using the dedicated Mission Specialist interface $(M=3.28, S D=1.16)$. These results suggest that mission trial $1(M=3.80, S D=0.74)$ and mission trial $2(M=4.28$, $S D=0.63$ ) had, on average, less than one half confidence rating point difference. There is also an average of less than one confidence rating point difference based on which interface was first used.

\section{E. Observations}

An analysis of the exploratory study results for a dedicated Mission Specialist interface resulted in five observations: viewing a mirrored display and instructing the Pilot and Navigator tended to result in more captured images, welldefined identification and image capture tasks resulted in similar results using both interfaces, similar levels of stress are experienced with both interfaces, similar visual feedback confusion was experienced using both interfaces, and more positive role empowerment was reported for the traditional approach. These observations suggested that with refinements, a dedicated Mission Specialist interface could be a useful tool for further exploring role performance in micro UAS.

Observation 1: Participants captured more images when viewing a mirrored display and instructing the Pilot and Navigator to control the payload camera and capture images. During the study, though time was emphasized as a limiting factor (i.e., 7-minute maximum flight time) and participants were asked only to capture a representative image, there was no penalty associated with capturing additional images. This led to multiple instances where participants using the traditional approach directed the Pilot and Navigator to perform exploratory 360-degree rotations of the UAV in order to capture several different images surrounding the train derailment. A similar observation did not occur with the dedicated interface where participants were more focused on controlling the payload camera. This difference could be due to the fact that giving instructions requires less effort of the participant, though it results in more action being taken by the Pilot and Navigator.

Observation 2: For identification and image capture tasks that were well-defined, participants provided satisfactory responses and captured images in a similar manner when using both interface approaches. Well-defined tasks included reading and capturing images of various numbers printed on train cars and identifying punctures, ruptures, and vents on an overturned tanker truck. For tasks that were not well-defined, participants captured more representative images when viewing the mirrored display and instructing the Pilot and Navigator to control the payload camera. Identification and image capture tasks that were not well-defined included identifying any hazardous product flows or possible exposure hazards associated with the derailment.

Observation 3: Participants experienced a similar level of stress as measured by average heart rate for both interfaces. The dedicated interface was not observed to induce any more stress than the traditional approach. Each of the participants had been trained as a specialized emergency responder to work in high-stress search and rescue situations; therefore, as realistic as the simulated train derailment was, it may not have fully replicated the conditions for which they are 
uniquely trained. Additionally, both types of interfaces used in the mission trial conditions represented a new experience to each of the participants, thus the non-significant level of stress recorded may have reflected the general lack of familiarity with both of the interface technologies.

Observation 4: Participants experienced a lack of adequate visual feedback when using both interface approaches. In both interface cases, each participant experienced at least one instance of difficulty with establishing payload camera status (i.e., extent of zoom or degree of tilt). For example, participants would attempt to zoom the payload camera in when the camera was already at maximum zoom. This suggests that payload camera status feedback should be provided visually to the Mission Specialist even though this feedback is not presently available to the Pilot and Navigator. This is especially important if the Mission Specialist is a member of an ad hoc human-robot team and does not have training similar to that of the Pilot and Navigator.

Observation 5: Participants reported more positive role empowerment when viewing a mirrored display and instructing the Pilot and Navigator to control the payload camera and capture images. Since role empowerment was reported as confidence in the ability to execute tasks with each interface, a possible cause of lower role empowerment may be latency. Each of the participants experienced some degree of latency in the payload camera controls when using the dedicated Mission Specialist interface. This was due in part to the technical limitations of an older UAV platform that was used for the study and may not be present in newer or different platforms. Conversely, the proprietary Pilot and Navigator controls allowed for faster and smoother operation of the payload camera. If the control scheme for the Pilot and Navigator could be better adapted to software-based control for a dedicated Mission Specialist interface, the latency issue would be likely be resolved.

\section{CONCLUSIONS}

To summarize, this exploratory study for a micro UAS Mission Specialist provided observations that when using a dedicated interface fewer images were captured, well-defined tasks resulted in a similar number of images captured, similar levels of stress were experienced, adequate visual feedback was not experienced, and less positive role empowerment was reported by the Mission Specialist than when viewing a mirrored display and instructing the Pilot and Navigator.

The fewer number of images captured when the Mission Specialist used the dedicated interface may depend on the cost associated with capturing images, which the exploratory study did not adequately address. More specifically, the amount of effort required to complete a task was not considered and may negatively impact the Pilot and Navigator role.

Well-defined tasks resulted in a similar number of correct identifications and images captured with both interfaces.
Future studies should focus on well-defined tasks for the evaluation of interfaces.

The levels of stress experienced by a Mission Specialist are expected to be similar, regardless of the type of interface used, and may depend on the training of the participant. An actual train derailment scenario (i.e., an ethnographic study) may result in different or higher levels of stress experienced by similar participants.

A lack of adequate visual feedback was experienced by all participants in this study. Future interfaces should incorporate visual feedback indicators for the payload camera status even though this information may not be accessible to the Pilot and Navigator.

Less positive role empowerment was reported when using a dedicated interface, which was likely due to latency in the controls that were used. Removing this confound may result in greater levels of confidence for users of a dedicated Mission Specialist interface.

This exploratory study suggests that more work is needed to understand user interface behavior for the Mission Specialist role in micro UAS. In the future it is anticipated that UAS technology, especially in the micro category, will require more software-oriented customization for general operation and human interaction; therefore, understanding how to optimize individual and team performance through HRI-informed role interfaces will be important for increasing performance and reducing manpower in micro UAS operations.

\section{ACKNOWLEDGMENTS}

This material is based upon work supported by the National Science Foundation under Grant No. IIS-1143713, EAGER: Shared Visual Common Ground in Human-Robot Interaction for Small Unmanned Aerial Systems. The authors would like to thank Chief David Martin and Mr. Clint Arnett from TEEX for their assistance with this study.

\section{REFERENCES}

[1] K. Robinston. (2011, August 18) "Darley Stinger drone helicopter gives firefighters eye in the sky," FireRescue1. [Online]. Available: http://www.firerescue1.com/fireproducts/communications/articles/1106762-Darley-Stinger-dronehelicopter-gives-firefighters-eye-in-the-sky

[2] P. Finn. (2011, January 23) "Domestic use of aerial drones by law enforcement likely to prompt privacy debate," The Washington Post. [Online]. Available: http://www.washingtonpost.com/national/domesticuse-of-aerial-drones-by-law-enforcement-likely-to-prompt-privacydebate/2011/01/22/ABLD0MR_story.html

[3] R. Post. (2011, July 27) "Micro-copter UAVs mapping landslide," GeoPrac.net. [Online]. Available: http://www.geoprac.net/geonewsmainmenu-63/65-geologic-hazards/1021-microcopter-uavs-mappinglandslide

[4] United States Air Force. "United States Air Force unmanned aircraft systems flight plan 2009-2047," [Online]. Available: http://www.defense.gov/dodcmsshare/briefingslide/339/090723-D6570C-001.pdf

[5] United States Army. "U.S. Army unmanned aircraft systems roadmap 2010-2035," [Online]. Available: http://www.fas.org/irp/program/collect/uas-army.pdf 
[6] Office of the United States Secretary of Defense. "Unmanned aircraft system roadmap 2005-2030," [Online]. Available: http://www.fas.org/irp/program/collect/uav_roadmap2005.pdf

[7] B. Gielow. (2012, February 2) "Congress sets 2015 deadline for unmanned aircraft systems to fly in the national airspace ," AUVSI News. [Online]. Available: http://www.auvsi.org/AUVSI/News/

[8] J. M. Peschel and R. R. Murphy. "Mission specialist interfaces in unmanned aerial systems," In Proceedings of the Sixth ACM/IEEE International Conference on Human-Robot Interaction, pp. 225-226, 2011.

[9] R. R. Murphy, K. S. Pratt, and J. L. Burke. "Crew roles and operational protocols for rotary-wing micro-UAVs in close urban environments,' In Proceedings of the Third ACM/IEEE International Conference on Human-Robot Interaction, pp. 73-80, 2008.

[10] A. P. Tvaryanas. "Human systems integration in remotely piloted aircraft operations," Aviation, Space, and Environmental Medicine, vol. 77, no. 12, pp. 1278-1282, 2006.

[11] B. Walters and M. J. Barnes. "Modeling the effects of crew size and crew fatigue on the control of tactical unmanned aerial vehicles (UAVs)", In Proceedings of the Winter Simulation Conference, pp. 920-924, 2000.

[12] A. Hobbs. "Unmanned aircraft systems," In E. Salas and D. Maurino, editors, Human Factors in Aviation, 2nd Edition, pp. 505-531. Elsevier, 2010.

[13] E. Clarkson and R. C. Arkin. "Applying heuristic evaluation to humanrobot interaction systems," FLAIRS Conference, pp. 44-49, 2007.

[14] G. Hoffman and C. Breazeal. "Collaboration in human-robot teams," In Proceedings of the First AIAA Intelligent Systems Technical Conference, pp. 18, 2004.

[15] R. R. Murphy and J. L. Burke. "From remote tool to shared roles," In IEEE Robotics and Automation Magazine, special issue on New Vistas and Challenges for Teleoperation, vol. 15, no. 4, pp. 39-49, 2008.

[16] D. D. Woods and E. Hollnagel, Joint Cognitive Systems: Patterns in Cognitive Systems Engineering. Boca Raton, FL: CRC Press, 2006.

[17] J. A. Jacko and A. Sears. The Human-Computer Interaction Handbook: Fundamentals, Evolving Technologies and Emerging Applications. Mahwah, NJ: Lawrence Erlbaum Associates, 2003.

[18] A. Dix, J. Finlay, G. Abowd and R. Beale. Human-Computer Interaction, 2nd Edition. London, England: Prentice Hall Europe, 1998.

[19] B. Schneiderman and C. Plaisant. Designing the User Interface: Strategies for Effective Human-Computer Interaction, 5th Edition. New York, NY: Addison-Wesley, 2010.

[20] H. Sharp, Y. Rogers and J. Preece. Interaction Design: Beyond HumanComputer Interaction, 2nd Edition. West Sussex, England: John Wiley \& Sons, Ltd., 2007.

[21] D. A. Norman. The Design of Everyday Things. New York, NY: Basic Books, 2002.

[22] S. Few. Information Dashboard Design: The Effective Visual Communication of Data. Sebastopol, CA: O'Reilly Media, Inc., 2006.

[23] M. R. Endsley, B. Bolte and D. G. Jones. Designing for Situation Awareness: An Approach to User-Centered Design. New York, NY: Taylor \& Francis, Inc., 2003.

[24] B. Mutlu, S. Osman, J. Forlizzi, J. Hodgins and S. Kiesler. "Task structure and user attributes as elements of human-robot interaction design," In Proceedings of the Fifteenth IEEE International Symposium on Robot and Human Interactive Communication, pp. 74-79, 2006.

[25] J. A. Adams. "Human-robot interaction design: Understanding user needs and requirements," In Proceedings of the Forty-Ninth Annual Meeting of the Human Factors and Ergonomics Society, pp. 447-451, 2006.

[26] A. Steinfeld, T. Fong, D. Kaber, M. Lewis, J. Scholtz, A. Schultz and M. Goodrich. "Common metrics for human-robot interaction," In Proceedings of the First ACM/IEEE International Conference on Human-Robot Interaction, pp. 33-40, 2006.

[27] M. A. Goodrich and D. R. Olsen, Jr. "Seven principles of efficient human-robot interaction," In Proceedings of the IEEE International Conference on Systems, Man and Cybernetics, vol. 4, pp. 3942-3948, 2003.

[28] J. M. Riley, L. D. Strater, S. L. Chappell, E. S. Connors and M. R. Endsley. "Situation awareness in human-robot interaction: Challenges and user interface requirements," In M. Barnes and F. Jentsch, editors, Human-Robot Interaction in Future Military Operations, pp. 171-192, 2010.

[29] T. Oron-Gilad and Y. Minkov. "Remotely operated vehicles (ROVs) from the bottom-up operational perspective," In M. Barnes and F. Jentsch, editors, Human-Robot Interaction in Future Military Operations, pp. $211-227,2010$

[30] Willow Garage. (2012) "OpenCV (Open Source Computer Vision) Wiki”. [Online]. Available: http://opencv.willowgarage.com

[31] C. L. Bethel and R. R. Murphy, "Review of human studies methods in HRI and recommendations," International Journal of Social Robotics, vol. 2, no. 4, pp. 347-359, 2010. 